\title{
Cómo organizar una escuela de padres sin morir en el intento
}

\author{
C. Núñez Jiménez \\ CS Trencadors, Mallorca. España.
}

\section{INTRODUCCIÓN}

Ayudar a crecer a un niño* o adolescente implica toda una tarea, un reto, una aventura apasionante. Sin embargo, en el día a día muchos padres se encuentran desconcertados y se enfrentan a la tarea de ayudar a crecer con muchas contradicciones.

Cuántas veces oímos "es que ahora los niños vienen enseñados", "es que todo depende de cómo los eduquen", "es que son el fiel reflejo de sus padres", "es que hoy son ellos los que mandan en casa, antes no era así... bastaba con que mi padre o mi madre me miraran" ¿Qué está pasando? ¿Es la educación? ¿Qué papel cumple el adulto?

La crianza de los hijos se inicia con gran ilusión pero también con muchas dudas, angustias, etc., frente a las cuales o bien se supone que "se sabe" por ser padre o madre o se supone que cualquiera puede opinar porque los padres "no saben".

Sin embargo, no siempre se es consciente de la necesidad de tener un espacio donde reflexionar acerca de esos temas y ante la propuesta de una escuela de padres se dan respuestas de todo tipo:

- ¿Para qué? Lo mejor es la observación y la intuición.
- Muy interesante, desde que supimos que esperábamos un hijo leemos revistas, libros, etc., al respecto.

- Interesante, nos formamos para todo menos para ser padres.

- Pero si entre extraescolares, colegio, etc., no tenemos tiempo.

- Creo que deberían ser obligatorias.

- Yo ya le he buscado un buen colegio. Allí ya le enseñarán todo.

\section{¿Y NUESTRO PUNTO DE VISTA?}

Entendemos que, en el marco de la sociabilidad actual, el proceso de crecer saludable está afectado tanto en su concepción como en la manera de llevarlo a cabo. ¿Qué es lo que desea un padre o una madre para su hijo? Muchas personas contestarán "que sea feliz", "que sea una buena persona". ¿Qué hay que hacer para conseguir esto? ¿Qué relación guarda la autonomía con ser feliz?

Favorecer el desarrollo de la autonomía en niños o adolescentes constituye un reto social del que deben ocuparse familias, profesionales e instituciones.

*Utilizamos la palabra niño refiriéndonos indistintamente al género femenino y masculino.

Cómo citar este artículo: Bueno Sánchez AM. Exploración de columna y cadera. Cómo manejar la escoliosis. Rev Pediatr Aten Primaria Supl. 2014;(23):119-25. 
Los procesos de crecer saludable se ven afectados; el desear las cosas de forma inmediata, la baja tolerancia a la frustración, el desprestigio de las normas, el no poner límites, dificultan los procesos de aprendizaje.

Los roles de padre y madre, la dificultad en la conciliación de la vida laboral, el consumismo, las prisas, dificultan los procesos de aprendizaje.

\section{PROGRAMACIÓN EN LA EDUCACIÓN PARA LA SALUD}

Cuando nos planteamos un proyecto de educación para la salud (EpS), no lo hacemos de forma improvisada, sino que debe estar planificado y organizado.

Los pasos de la programación de la EpS son:

0. Justificación.

1. Análisis de situación.

2. Objetivos y contenidos.

3. Actividades, métodos, técnicas.

4. Evaluación.

\section{Justificación}

La primera fase del proceso de planificación consiste en la identificación de las necesidades que pueden ser susceptibles de actividades educativas. En esta etapa se realiza un análisis, con el objetivo de conocer la situación en la que encuentra la población.

Una vez identificadas las necesidades, les daremos un orden de prioridad. Para ello tendremos en cuenta las siguientes preguntas:

- ¿Cuántos tienen el problema o necesidad?

- ¿A cuántos les preocupa?

- ¿Cuántos están dispuestos a aprender?

- ¿Los profesionales sanitarios están en condiciones y tienen capacidad para orientar el aprendizaje? ¿Con qué profesionales de otros sectores podemos contar? (matrona, trabajador social, psicólogo, algún padre o madre...).
En nuestro caso, en este punto deberemos definir con qué población queremos trabajar, con familias de adolescentes, con familias de niños de 0-12 meses, de qué edad, familias inmigrantes, familias con niños con alguna discapacidad...

\section{Análisis de situación}

Antes de plantear cualquier tipo de intervención educativa individual o grupal es necesario conocer de dónde partimos. Esto nos permitirá plantear objetivos y actividades lo más adecuadas posibles a las necesidades y realidades de la persona o colectivo con la que vayamos a trabajar.

Debemos conocer la población diana. Se trata de definir cuál es el grupo sobre el que vamos a actuar. Cuanto mejor definamos este, mejor vamos a poder diseñar una intervención apropiada a sus características. Características que tienen que ver con su dimensión biológica, como puede ser el sexo y la edad; dimensión económica, como puede ser el bajo, medio o alto nivel económico; dimensión educativa previa: la exposición al riesgo, si es bajo, medio o alto, y con la motivación-disposición que tienen para mejorar-solucionar el problema.

Esto nos servirá para ver cómo debemos sensibilizar, qué métodos podemos utilizar, cómo haremos la captación.

Hay que identificar los comportamientos y los factores relacionados.

Se trata de algunos datos sobre conocimientos, creencias, valores, actitudes, habilidades, comportamientos, y los factores que se relacionan con ellos.

Esta información permitirá priorizar los objetivos y los contenidos a trabajar.

Para llevar a cabo la recogida de datos utilizaremos encuestas, revisión bibliográfica, entrevistas, grupos de debate.

La recopilación de datos se llevará a cabo antes de la constitución del grupo.

Hay que tener en cuenta que la utilización de una metodología activa y participativa a lo largo del proceso educativo proporciona una información 
continua sobre los comportamientos de la persona o grupo y de los factores relacionados. Por ello, el análisis de situación se reelabora día a día.

\section{Objetivos y contenidos}

Los objetivos son de dos tipos:

\section{Generales}

- Expresan la finalidad del proyecto.

- No son evaluables.

"Favorecer el desarrollo integral de los niños y niñas y sus familias, mejorando las capacidades parentales."

\section{Especificos}

- Surgen del análisis de situación.

- Son evaluables.

- Deben hacer referencia a las tres áreas del aprendizaje (cognitiva, afectiva y habilidades).

"Que las familias que acudan al taller reorganicen e incrementen los conocimientos acerca del desarrollo evolutivo de sus hijos."

"Que las familias expresen sus vivencias, experiencias y emociones acerca del rol de padre/madre."

"Que las familias desarrollen habilidades acerca de dar una respuesta adecuada frente a sintomas de alarma."

Respecto a los contenidos a trabajar, los aportará el educador y todas las personas que participan en el taller. Al igual que los objetivos, el taller será más rico si los contenidos abarcan las tres áreas.

En cuanto a los contenidos, en la bibliografía consultada ${ }^{1-6}$ hemos encontrado grandes bloques a trabajar:

\section{Ser padre/madre hoy. Rol de padre/madre en mi proyecto de vida}

¿Qué significa hoy en día ser padre o madre? ¿Cómo desempeñar ese rol de forma óptima? ¿Qué roles tengo y cuales me gustaría tener?
Los objetivos a la hora de trabajar este contenido podrían ser:

- Que los padres expresen, compartan, reorganicen sus experiencias, vivencias respecto a la maternidad/paternidad.

- Que analicen ese nuevo rol adquirido.

- Que reflexiones sobre cómo se integra el rol de padre/madre en su proyecto de vida.

Ejemplos de dinámicas:

- Tormenta de ideas sobre aspectos satisfactorios de mi vida desde el nacimiento de mi bebé.

- Rejilla "Comenta los cambios que te han supuesto como madre/padre al entrar tu hijo en la adolescencia: aspectos positivos/dificultades".

- Análisis de vídeo (series como Cuéntame, Los Serrano). Analizar cómo ha cambiado el rol de padre/madre a través del tiempo.

- Análisis de publicidad (http://youtu.be/LW LGS4M18g).

\section{Cuidar a nuestros hijos (hábitos saludables,} vacunación, accidentes, enfermedades...)

Objetivos:

- Ejercitar habilidades que permitan elaborar una dieta saludable.

- Que conozcan los síntomas de alarma de las patologías más frecuentes en los niños.

- Que sean capaces de actuar ante un accidente doméstico.

Dinámicas:

- Trabajar la elaboración de un menú semanal.

- Falsos mitos respecto a la alimentación/frases verdadero-falso.

- Role playing acerca de cómo actuar ante un accidente, actuación ante la fiebre.

- Análisis de víeo (http://youtu.be/lkNJ4kEp PBM).

\section{Educar a nuestros niños: aceptación, autoestima, autoridad. Crianza sin violencia}

¿Qué es educar? ¿Cómo nos educaron? Estilos educativos. 
Objetivos:

- Comprender y reflexionar sobre qué es educar, el rol de educador.

- Elaborar elementos que instrumenten en el ejercicio de la autoridad y puesta de límites.

Dinámicas:

- Cómo nos educaron: recordar cómo fue nuestra educación (experiencias positivas y negativas). Posteriormente reflexionar acerca de los cambios que se han producido desde entonces y a qué pueden deberse.

- Debate a dos bandas; una parte del grupo defiende un estilo autoritario y otra está en contra.

- Análisis de casos (historias sobre aspectos cotidianos: niño que no se quiere ir solo a la cama, llama a sus padres en múltiples ocasiones; niña que cuando no le dan lo que quiere se tira al suelo y se pone a gritar...).

\section{Conocer mejor a nuestros hijos: desarrollo evolutivo}

¿Qué características tienen los niños en las distintas edades? ¿Qué habilidades tiene mi hijo? Valoración del juego como instrumento para conocerles y relacionarnos mejor.

Objetivo:

- Comprender las características que corresponden a cada etapa de la vida de nuestros hijos.

Dinámicas:

- Frases incompletas.

- Philips 6:6 sobre las características evolutivas a determinadas edades.

- Lección participada sobre las distintas características en el desarrollo evolutivo.

- Falsos mitos; presentaremos una lista de ideas erróneas acerca del desarrollo evolutivo de los niños; en grupos pequeños trabajarán esos mitos. Luego en grupo grande se llevará a cabo una puesta en común.

\section{Mejorar la relación educativa: observación y escucha, comunicación, manejo de conflictos}

¿Cómo me comunico con mis hijos y cómo se comunican mis hijos conmigo? En este bloque se trabajan sobre todo habilidades: comunicación, asertividad, manejo de conflictos y negociación, pero también los aspectos cognitivos y emocionales que subyacen en esas habilidades.

Objetivos:

- Que expresen, reflexionen acerca de los conflictos que surgen con sus hijos.

- Que desarrollen habilidades para el uso de mensajes yo.

Dinámicas:

- Role playing con una situación frecuente ("la familia ha decidido irse de vacaciones al pueblo y al comunicárselo al hijo este ya tenía plan para pasar las vacaciones con sus amigos").

- Estudio de viñetas ${ }^{7}$.

\section{Técnicas/métodos/actividades}

Los métodos y técnicas que se utilizan al hacer EpS no son solo lecciones. Se utilizan una combinación de métodos de distintos tipos: casos, lecciones, análisis de películas, publicidad, simulaciones, rejillas... Están basados en el aprendizaje significativo, esto es, son métodos inductivos (que parten de la experiencia de los educandos) y activos (que implican a las personas).

Las actividades educativas se agrupan en sesiones. Cada actividad se diseñará teniendo en cuenta el fin de aprendizaje que se quiera.

Existen cuatro grandes grupos de técnicas, dependiendo del objetivo de aprendizaje (Tabla 1).

A la hora de definir las técnicas a utilizar, es de gran ayuda la elaboración de un cronograma; en este, además de la técnica a utilizar, pondremos el tiempo y la agrupación (si se va a trabajar de forma individual, en parejas, grupo pequeño o grande). 


\begin{tabular}{|l|l|l|}
\hline \multicolumn{1}{|c|}{ Tabla 1. Técnicas } & \multicolumn{1}{c|}{ Objetivo aprendizaje } & \multicolumn{1}{c|}{ Tipos } \\
\hline Investigación en el aula & $\begin{array}{l}\text { Cuando queremos conocer la } \\
\text { realidad del grupo, sus creencias, } \\
\text { valores, conocimientos }\end{array}$ & $\begin{array}{l}\text { Tormenta de ideas } \\
\text { Rejilla, Philips 6/6 } \\
\text { Frases incompletas } \\
\text { Cuestionario, cuchicheo }\end{array}$ \\
\hline Análisis & Profundizar & $\begin{array}{l}\text { Caso, análisis vídeos } \\
\text { Películas, anuncios, relatos }\end{array}$ \\
\hline Expositivas & $\begin{array}{l}\text { Reorganizar, contrastar } \\
\text { conocimientos }\end{array}$ & $\begin{array}{l}\text { Lección participada } \\
\text { Panel de expertos, mesa redonda }\end{array}$ \\
\hline Desarrollo de habilidades & $\begin{array}{l}\text { Entrenar en habilidades concretas y } \\
\text { desarrollar capacidad de actuar, de } \\
\text { comportarse en situaciones reales }\end{array}$ & Role playing \\
\hline
\end{tabular}

\section{Evaluación}

La evaluación tiene como objetivo obtener información que permita adecuar el proceso de enseñanza al progreso real en la construcción de aprendizajes, adecuando el diseño y desarrollo de la programación establecida a las necesidades y logros detectados.

En la evaluación debemos considerar:

- ¿Quién evalúa?

- La evaluación la realiza tanto el educador como los educandos, así como observadores externos.

- ¿Qué se evalúa?

- Los resultados; el grado de consecución de los mismos.

- El proceso; idoneidad de las actividades, asistencia, participación, grado consecución objetivos.

- La estructura; adecuación de los recursos, local. Duración sesiones recursos personales.

- ¿Cómo se evalúa?

- De forma continuada, a lo largo de todo el proceso.

\section{ROL DEL EDUCADOR}

En la enseñanza bidireccional el educador es un guía, un facilitador. En la EpS el importante es el grupo.
- Funciones: existen unas funciones asociadas al rol de educador; estas son una buena preparación previa y, a lo largo del taller, la gestión de la tarea, de los recursos y del tiempo.

\section{- Actitudes:}

- Congruencia: el educador debe ser coherente, auténtico, genuino.

- Aceptación: debe aceptar a los educandos tal como son; no se trata de estar de acuerdo ni de compartir sus puntos de vista, pero tampoco de intentar cambiar ni manipular.

- Valoración positiva.

- Empatía: "ponerse en el lugar de".

- Habilidades y recursos: escucha activa, asertividad, buen comunicador, autocontrol emocional; debe saber resumir y devolver, ayudar a pensar, reforzar positivamente, negociar y resolver conflictos.

Las actitudes del educador se relacionan con el clima del aula. Existen climas defensivos o tolerantes dependiendo del proceso de comunicación entre docente y educandos y entre estos (Tabla 2).

Lo más adecuado que el docente puede hacer para no gestionar las relaciones entre los participantes pero si crear un clima útil al aprendizaje es reducir el grado de defensa en la comunicación entre él y los educandos. 


\begin{tabular}{|c|c|}
\hline Climas defensivos & Climas tolerantes \\
\hline Valoración & Descripción \\
\hline Control & Orientación del problema \\
\hline Rigidez & Espontaneidad \\
\hline Distancia & Relación de iguales \\
\hline Superioridad & Aprendizaje mutuo \\
\hline Certeza & Intentar conocer otros puntos de vista \\
\hline Dependencia & Autoridad de servicio \\
\hline
\end{tabular}

\section{CLAVES PARA MOTIVAR}

\section{Captar la atención de los discentes}

- Sacarle el máximo partido a los audiovisuales (dibujos, películas, canciones...).

- Echarle un poco de teatro a la clase (dramatizar, cambiar de ritmo...).

- Ayuda a captar la atención:

- Hablar con voz clara y volumen suficiente.

- Uso de medios audiovisuales que se vean y oigan bien.

- Disposición de los alumnos en círculo.

- Repetir y resumir las explicaciones.

- Evitar el ruido ambiental.

- Iniciar el tema comentando algún acontecimiento o noticia de actualidad.

- Renovar cada cierto tiempo nuestros apuntes, los recursos, las técnicas.

- Hacer ver al grupo la utilidad del tema que tratamos.

- Utilizar muchos ejemplos.

- Crear expectación.

Hacerles ver la actualidad e importancia de lo que estamos haciendo

- Debemos procurar conocer al grupo desde el comienzo del curso.

- Hacerles ver lo importante que resulta conocer lo mejor posible cada uno de los temas o cuestiones que estamos tratando.

- Adaptar nuestra programación al grupo concreto.

\section{Fomentar la participación en todo momento}

- Estimular con palabras, todo tipo de participación en el alumnado.

- Contar con ellos para resolver problemas metodológicos.

- Estar pendiente de las personas que no participan.

\section{Dosificar el esfuerzo a realizar para lograr el objetivo}

- Dividir el proceso en etapas concretas y perfectamente superables.

- No dedicar toda la sesión a la misma actividad.

- Corregir cuando sea necesario.

- No aplastar al caído.

\section{Estimular su iniciativa, creatividad y toma de decisiones}

- Plantearles problemas a resolver.

- Aplaudir y elogiar su iniciativa y creatividad.

- Parar en seco los abucheos y risitas que suelen acompañar a las iniciativas de algún educando.

\section{Indicar desde el inicio el proceso a seguir} y el objetivo a lograr

"Al que no sabe adónde va, todos los vientos le son contrarios."

- Programar al principio del curso tanto los objetivos a superar como los caminos para lograrlo.

- Repasar con los alumnos, de vez en cuando, a medida que va transcurriendo el curso, el camino recorrido provocando para que relacionen lo novedoso con lo ya aprendido. 


\section{Estimular su natural espíritu de superación} y cooperación

"La gracia está en mejorar."

- Procurar que los alumnos reflexionen sobre sus propias facultades físicas y psíquicas.

- Presentar la evaluación de cualquier actividad humana como algo básico para poder mejorarla.

- Evitar en todo momento la comparación entre unos y otros.

\section{Proponer y convencer, nunca imponer}

- Dialogar mucho con los educandos.

- Evaluar juntos los resultados obtenidos tras un determinado periodo de aprendizaje.

\section{Predicar con el ejemplo}

"Nadie da lo que no tiene."

\section{BIBLIOGRAFÍA}

1. Pérez Jarauta MJ, Echauri Ozcoidi M, Ancizu Irure E, Chocarro San Martín J. Manual de Educación para la Salud. Gobierno de Navarra. Instituto de Salud Pública. 2006 [en línea]. Disponible en: www.cfnavarra. es/isp/promocion

2. Ansa A, Begue R, Cabodevilla I, Echauri M, Encaje S, Montero P, et al. Ayudar a crecer. Guía educativa para trabajar con grupos de padres y madres. Pamplona. Gobierno de Navarra. 2004 [en línea]. Disponible en: www.cfnavarra.es/isp/promocion

3. Ansa A, Begue R, Cabodevilla I, Echauri M, Encaje S, Montero P, et al. Guía de salud y desarrollo personal para trabajar con adolescentes [en línea]. Disponible en: www.cfnavarra.es/isp/promocion
- No exigir a nuestros alumnos algo que nosotros nunca hemos hecho (y, a lo peor, somos incapaces de hacer).

- Coherencia entre lo que se dice y lo que se hace.

- Reconocer los errores cuando los haya y pedir disculpas (también nosotros somos imperfectos).

\section{Emplear dinámicas diversas}

\section{CONFLICTO DE INTERESES}

La autora declara no presentar conflictos de intereses en relación con la preparación y publicación de este artículo.

\section{ABREVIATURAS}

EpS: educación para la salud.

4. González R, Martín S, Román Y. Queriendo se entiende la familia. Guía de intervención sobre parentalidad positiva para profesionales. Save the children. 2013 [en línea]. Disponible en http://www.savethe children.es/quieroquetequiero

5. González R, Román Y. ¿Quién te quiere a ti? Guía para padres y madres: cómo educar en positivo. Save the children. 2012 [en línea]. Disponible en: http://www. savethechildren.es/quieroquetequiero

6. Martínez González MA. Guía para el desarrollo de competencias emocionales, educativas y parentales. Ministerio de Sanidad y Política social. 2009.

7. Faber A, Mazlish E. Cómo hablar para que sus hijos le escuchen y cómo escuchar para que sus hijos le hablen. Barcelona: Medici; 2007. 